\title{
Analysis of Defects of PV Solar Modules Using Deep Level Transient Spectroscopy. Feasability and Limits.
}

\author{
Jean Zaraket ${ }^{1,2, a)}$, Ninel Kokanyan ${ }^{3}$, Michel Aillerie ${ }^{2}$, and Chafic Salame ${ }^{1,4}$ \\ ${ }^{I}$ CEER, Faculty of Sciences II, Lebanese University, B.P 90656 Jdeidet El Mten, Lebanon \\ ${ }^{2}$ Université de Lorraine, CentraleSupélec, LMOPS, F-57000 Metz, France \\ ${ }^{3}$ CentraleSupélec, Université de Lorraine, LMOPS, F-57000 Metz, France \\ ${ }^{4}$ CNRSL, National Council for Scientific Research, Beirut, Lebanon \\ Corresponding author: jean.zaraket@hotmail.com
}

\begin{abstract}
In a photovoltaic solar module, temperature and shading variations or non uniform illumination but also any recombination of interconnection failure, cell failure are at the origin of local hot spots, variations in the photocurrent or mismatches from cell to another cell, aging and local deterioration of the modules with damages that can be irreversible. To analyze the intrinsic origin and the behavior of the damage phenomena with electrical and thermal stresses, a Deep Level Transient Spectroscopy, DLTS, spectrometer based on a double box-car system is used to characterize the parameters of PV modules under external stressed parameters. Thus, based on DLTS spectroscopy measurements, this, work discusses and analyzes the nature of defects present in PV solar modules. We performed series of experimental measurements after various reverse currents injected for stress, at various temperature in monocrystalline silicon commercial modules. To avoid additional contribution of the photovurrent, electrical and thermal stresses were created in dark into the solar module structure and DLTS measurements were performed before and after in dark conditions for several common periods. Experimental results and analysis point the relation between the parameters of defect obtained by DLTS measurements and the electrical parameters of PV module i.e. the increase of the diffusion current, the low value of shunt resistance and the high value of the ideality factor $(\mathrm{A}>1)$. The optimum results that can be achieved with the DLTS technique in the characterization of PV modules were discussed based on previous results published on stressed PV modules obtained by more classical methods. As confirmed by DLTS, originally used for this kind of characterisation, the main results show the huge influence of the working external conditions of PV solar modules in real conditions. This contribution points also the limitation of the DLTS technique for the characterization of complex electronic systems as PV panels.
\end{abstract}

Keywords: Solar cell/module, Electrical Stress, Thermal Stress, Semiconductor, Defects, DLTS measurement

\section{INTRODUCTION}

All crystalline semiconductors contain metallic impurities and structural defects due to the component processing, the semiconductor growth, and the operating conditions that have a huge influence on the concentration of defects. These impurities and defects induce additional energy levels in the band gap assuming electrons exchange with the valence and conduction band, and for that reason are called "recombination centers". It is also to note that the surface of a material is the place where the periodicity of the crystal lattice is interrupted and the recombination of the carriers is seen not only in the volume of the semiconductor but also at the surface. The number and localization of defects modify the conduction effects and straightforwardly influe the performance of semiconductor devices based on conduction as photovoltaic modules or numerous types of sensors.[1,2]

The energy levels near to the conduction band or near the valence band level, correspond to doping impurities of $\mathrm{N}$ or P types, respectively. When levels corresponding to defects or impurities are close to the center of the bandgap, these levels are deep traps. In case of photovoltaic (PV) cells or modules, these defects can capture photogenerated charge carriers through the photovoltaic conversion process, thus reducing the output current, the open circuit voltage and finally the performance of PV cells.

A performant tool to characterized the defects in the bad gap of single semi-conductor juntions is the the Deep Level Transient Spectroscopy (DLTS). DLTS technique was developed in 1974 by DV Lang [3], to investigate electronic "deep" levels in the space of semiconductor structures load, dedicated to be only used for junction PN or Schottky barriers. The DLTS technique relies on the measurement of transients capacitance resulting from the relaxation process after electrical excitation, characterized as a function of temperature for discrimination of the various 
contributions achieving in the capacitance. The defects will be filled during an initial pulse applied to the junction then emptying when the voltage pulse is removed. The difference between two transient points at a given temperature forms the DLTS signals. The analysis of these signals, with the temperature makes it possible, via the Arrhenius law to determine the activation energies and the capture cross sections of the defects.

This DLTS technique is a complementary performant method of indirect measurements of semiconductor junction defects, as for example, techniques used in the Ref. [4-6] and [7, 8] based on I(V) and C(V) characterizations. Thus, the DLTS technique is one of the most performant techniques for characterizations of internal defects in semiconductor juntions [9-11].

Thus, applied to photovoltaic engineering, the DLTS analysis of Si-based solar modules is expected to give interesting information about the energy levels and the corresponding defects present in the substrate. This is an important step to study the defects caused, initialy by the manufacturing process, or during the life of the module, as this induced by the thermal and electrical stresses appearing from an irregular shadding, mistmatches between panels or hot-spots created by dust and objects on the surface.

The experiments presented in this contribution concern, by using a DLTS measurement, the analysis of the defects in a PV junction and their influence on the efficiency of the PV cell before and after electrical and thermal stresses. The implementation of the DLTS technique, mainly its experimental possibilities offered thanks to its highperformance electronics associated with a double box-car system, is, to our knowledge, the first time reported in the literature for the characterization of complex PV modules. To discriminate the influence of the photo-current with the specific effect generated by the stress, and specific to it, this stress was induced with the module in dark conditions. To simulate a damaging reverse bias affecting the module over a certain period of time, the reverse current and temperature in dark impose a high level of electric and thermal stress in the opposite electron flow direction of the solar module. Results and discussions offered aim to contribute to material, performance and efficiency of photovoltaic solar module/cell research. Finally, within the original results in PV module characterization obtained in this work, a discussion concerning the limit of the DLTS technique for the charaterisation of complexe semiconductor elements will be also consider to conclude this contribution.

\section{FUNDAMENTALS}

For the studying of traps in semiconductors, the Deep Level Transient Spectroscopy (DLTS) [12] is a technique frequently used. This method is based on the determination of the characteristics of trapped charges after their injection into the semiconductor junction by an external applied a voltage. The filling, then the emptying of the traps produce a variation of charges in the semiconductor resulting a variation in the capacitance of the diode. It is particularly to study the traps in junction devices such as Schottky diodes or classic diodes PN. These traps are generally deeper than the donor or acceptor levels. [13] The procedure of measurement was showed in figure 1:

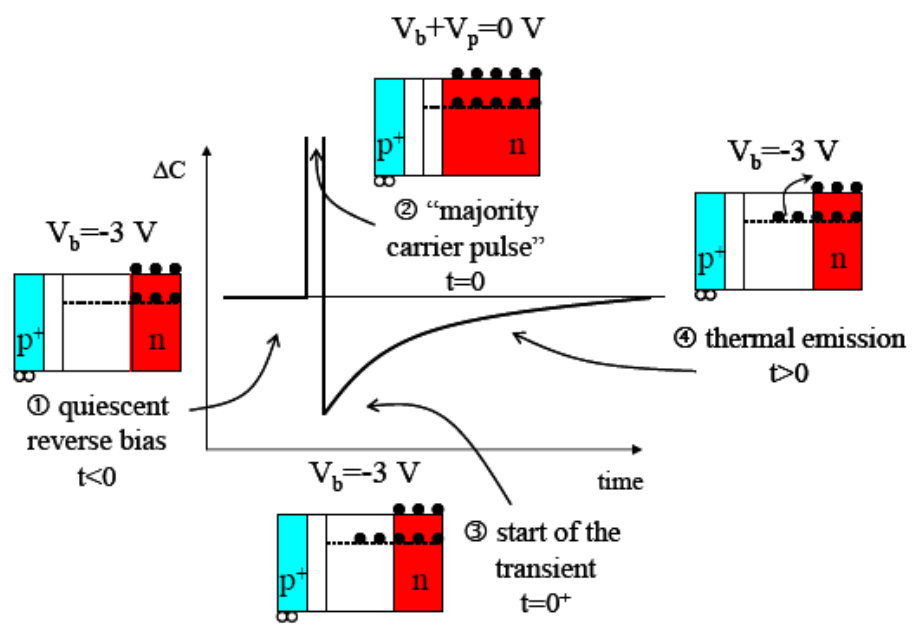

FIGURE 1. Differents phases of DLTS measurement [14] 
The theoretical study of the DLTS fundamentals and the equations below necessary for the self-consistency of this contribution are developed and summarized from the studies published in Refs [11-16].

1- We applied a reverse voltage $\mathrm{V}_{\mathrm{b}}$ at the junction. The ZCE is maximized, the trap level is empty inside of the ZCE, the capacity of the junction has a constant value which is determined by the equation:

$$
\frac{1}{C^{2}}=\frac{2\left(\emptyset_{i}-V_{a}\right)}{\varepsilon_{S} q N_{D}}
$$

2- We applied a voltage pulse ( $V_{p}$ for pulse and $\tau_{p}$ for duration) and as a maximum, $V_{b}+V_{p}=0$. This is called pulse majority because only electrons are injected. In this case, the width of the ZCE is reduced and the capacity increases.

3- As shown in the figure above, the voltage pulse is removed. This is the beginning of the transitional capacity (in this case the measurement started) because the component back to the initial level $\mathrm{V}_{\mathrm{r}}$ reverse bias. The traps are emptied gradually of captured charges capacity then amounts to a level below its original level. We obtained the equation:

$$
C=\left(\frac{\varepsilon_{S} q N_{e f f}}{2\left(\emptyset_{i}-V_{b}\right)}\right)^{\frac{1}{2}}
$$

4- Since the transient variation of the capacitance depends on the temperature, the method developed by Lang treats the transient part recorded. We can obtein the DLTS spectrum and signal characteristics extracted from the defect parameters (activation energies (Ec - Et) and the capture cross sections $\sigma$ ).[14]

In figure 2, we show the different phases presented above in phase 3 for the two moments of measurements.

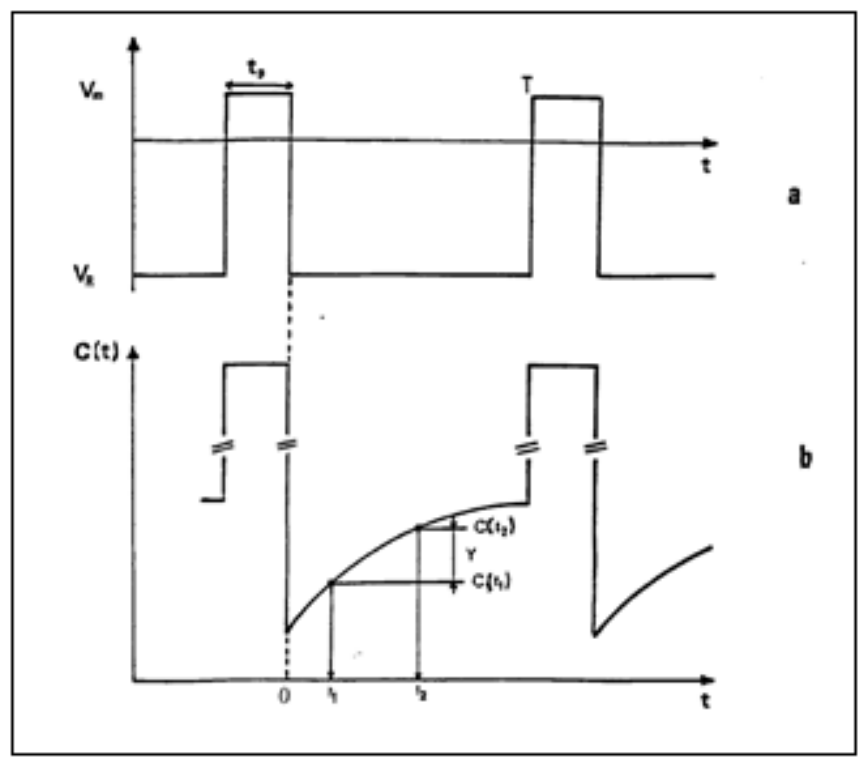

FIGURE 2. Diagram illustrating the phases of the DLTS measurement of (a) polarization voltages; (b) capacity transient. [15]

\section{The DLTS procedure}

The Lang's method is usually used to analyze DLTS signals deduced from measuring the variation of capacitance in the junction induced by the application of a voltage step. In this method, the capacitance variation $\Delta \mathrm{C}$ as a function 
of temperature was measured between two instants $t_{1}$ and $t_{2}$. Then we have to determine the variation of $\Delta \mathrm{C}$ for a given time pair $t_{1}$ and $t_{2}$. For this given time interval, we obtain a normalized DLTS signal as shown in figure 3, we obtain a maximum value of capacitance variation $\Delta \mathrm{C}_{\max }$ for a given temperature $\mathrm{T}_{\max }$. The characteristic of the defects [11] is given by the equation:

$$
\mathrm{DLTS}=\Delta \mathrm{C}=\mathrm{C}\left(\mathrm{t}_{2}\right)-\mathrm{C}\left(\mathrm{t}_{1}\right)=\left[\exp \left(-e_{n} t_{2}\right)-\left(-e_{n} t_{1}\right)\right]
$$

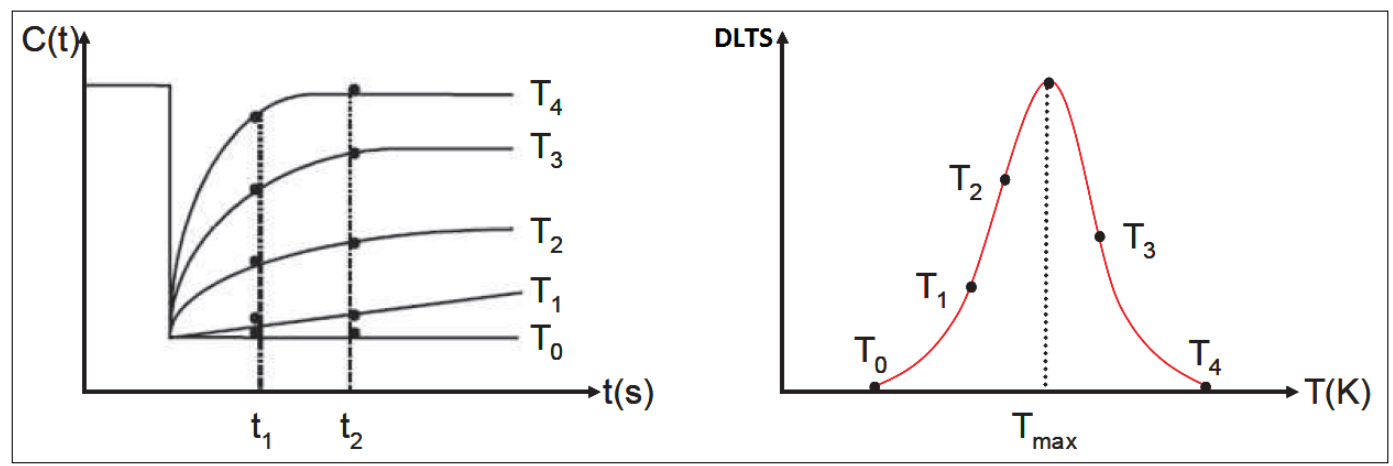

FIGURE 3. Evolution of the capacity transient and the DLTS signal $(\Delta \mathrm{C})$ vs the temperature, in this example $\mathrm{T}_{4}>\mathrm{T}_{3}>\ldots>\mathrm{T}_{0}[11]$

When the temperature is lower than $T_{\max }$, the emission of trapped electrons is made in a much lower time than $t_{2}$. But when the temperature is higher than $T_{\max }$, the emission of trapped electrons is made in a much lower time than $t_{1}$. Then, no variation of capacity is observed between $t_{1}$ and $t_{2}$ for low and high temperatures. On the other hand, in the observation between $t_{1}$ and $t_{2}$, it is possible to deduce from equation 3 the transmission speed corresponding to the DLTS signal maximum at the $\mathrm{T}_{\max }$ point. At this point $\mathrm{d} \Delta \mathrm{C} / \mathrm{dT}=0$ which can be decomposed in the following way $\left(\mathrm{d} \Delta \mathrm{C} / \mathrm{de}_{\mathrm{n}}\right)\left(\mathrm{de}_{\mathrm{n}} / \mathrm{dT}\right)=0$. The equation above is written $\left(\mathrm{de}_{\mathrm{n}} / \mathrm{dT}\right)=0$ and we get the reverse of the emission rate, i.e. the emission speed, $e_{n}=\ln \left[\left(t_{2} / t_{1}\right) /\left(t_{2}-t_{1}\right)\right]$.

The position of this maximum depends on the pairs of values of $t_{1}$ and $t_{2}$ chosen for recording the capacity. But whatever the pair $\left(t_{1}, t_{2}\right)$ chosen, the position of $T_{\max }$ and the amplitude of the DLTS signal at this temperature will depend on the level of the trap. [11]

For different pairs $t_{1}$ and $t_{2}$, we will obtain different DLTS signals with different values $\left(e_{n}, T_{\max }\right)$. From these parameters we can draw the Arrhenius diagram to determine the parameters.

Figure 4 represents an example of Arrhenius line to calculate the parameters such as the activation energy and the capture cross sections.

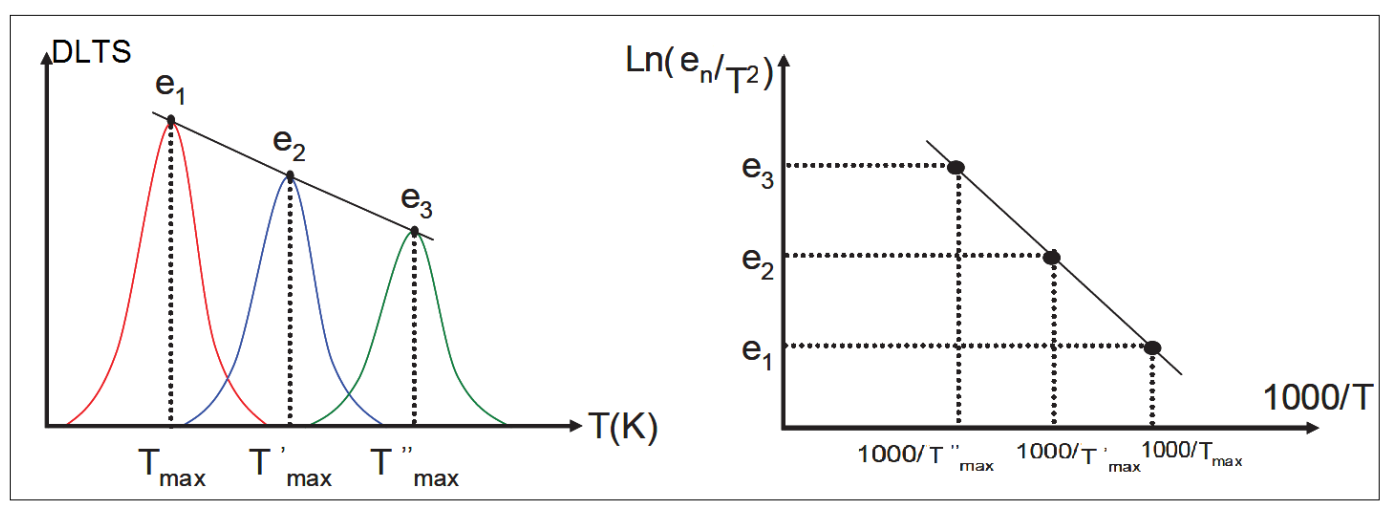

FIGURE 4. The Arrhenius line by the Lang's method [3]

The activation energy $E_{a}$ and the cross section of capture $\sigma$ can be determined by the expression of the electron emission rate $\mathrm{e}_{\mathrm{n}}$. By the relation [3]: 


$$
e_{n}(T)=A T^{2} \exp \left(-\frac{E_{a}}{K T}\right) \quad \text { and } \quad A=\frac{4 \sqrt{6} \sigma k^{2} \pi^{\frac{3}{2}} m^{*}}{h^{3}}
$$

$\mathrm{T}$ is the absolute temperature, $\mathrm{E}_{\mathrm{a}}$ : activation energy of the trap, $\sigma$ : cross section of capture, $\mathrm{K}$ is Boltzmann's constant, $\mathrm{m}$ *: effective mass of the electron, h: Planck's constant.

The plot of $\ln \left(\frac{e_{n}}{T^{2}}\right)$ as a function of $1 / \mathrm{kT}$ for the pairs of values $\mathrm{e}_{\mathrm{ni}}$ and $\mathrm{T}$ is carried out by the relation [13]:

$$
\ln \left(\frac{e_{n}}{T^{2}}\right)=\ln (A)-\frac{E_{a}}{K T}
$$

This plot corresponds to a straight line, called the Arrhenius line, whose slope is equal to the activation energy of the defect. And the intersection between the ordinate and the origin was equal to $\ln (\mathrm{A})$, makes to calculate the capture cross sections. In silicon cases, we have the following relation: $-\ln (\mathrm{A})=\ln \sigma+49,031$. [16]

\section{The Five Points Method}

The Lang's method presented above extracts the temperature $T_{\max }$ and the emission speed into the maximum value of the DLTS signal. In order to draw the Arrhenius line with a high number of points, it is possible to extract the temperature $T_{i}$ and the emission speed $e_{n i}$ in five particular points $(i=1 \ldots 5)$ of each DLTS peak. These five points are chosen as the extremum, the two points at $1 / 2$ of the maximum amplitude and the two points at $3 / 4$ of the maximum amplitude.

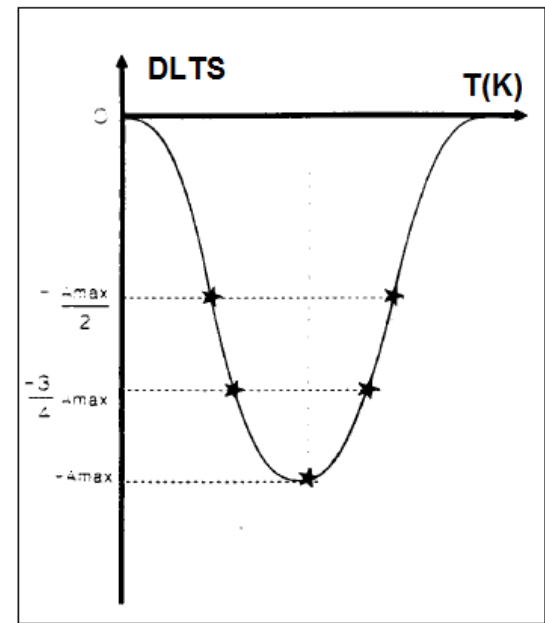

FIGURE 5. The choice of five points by the DLTS signal

In order to explain this method, we will present the calculations by fixing the ratio $\alpha=\frac{t_{2}}{t_{1}}=2$ making to set the maximum value of the parameter $\mathrm{A}$ to $\mathrm{A}_{\max }=\mathrm{A} / 4$ determined from the value of the maximum amplitude of the peak. This value is related to the equation 3 of the DLTS signal, we then obtain the equation:

$$
\begin{aligned}
& \left.\mathrm{A}_{\max }=\mid \mathrm{A}\left[\exp \left(-\operatorname{Ln}\left(\frac{t_{2}}{t_{1}}\right) / \frac{1-t_{1}}{t_{2}}\right)\right)-\exp \left(-\operatorname{Ln}\left(\frac{t_{2}}{t_{1}}\right) / \frac{t_{2}}{t_{1}-1}\right)\right] \mid \\
& \mathrm{A}_{\max }=\left|\mathrm{A}\left[\exp \left(\frac{-\operatorname{Ln}(\alpha)}{1-\alpha^{-1}}\right)\right)-\exp \left(\frac{-\operatorname{Ln}(\alpha)}{\alpha-1}\right]\right|
\end{aligned}
$$


The first step of the method is, from the equation 3 , to determine the emission speed $\mathrm{e}_{\mathrm{n} 3}$ corresponding to the maximum amplitude as proposed by Lang in his method. In the description of the DLTS method, we have previously presented the calculation of the emission speeds at this point which is obtained as a solution of the equation $\mathrm{d} \Delta \mathrm{C} / \mathrm{dT}=0$, i.e. $\mathrm{d} \Delta \mathrm{C} / \mathrm{d} \mathrm{e}_{\mathrm{n}}=0$. This particular value of the emission speed $\mathrm{e}_{\mathrm{n} 3}$, solution of this equation is thus found equal to $\mathrm{e}_{\mathrm{n} 3}=\ln \left(\frac{t_{2}}{t_{1}}\right) /\left(\mathrm{t}_{2}-\mathrm{t}_{1}\right)=0.693 / \mathrm{t}_{1}$. This value corresponds to the value of the maximum emission speed proposed in Lang's method. The emission speeds $\mathrm{e}_{\mathrm{n} 1}$ and $\mathrm{e}_{\mathrm{n} 5}$, can be calculated. They are solutions of the equation 3 when $\Delta \mathrm{C}=-\mathrm{A}_{\max } / 2=-\mathrm{A} / 8$ which makes it possible to write $\mathrm{x}^{2}-\mathrm{x}+1 / 8=0$, with $\mathrm{x}=\exp \left(-\mathrm{e}_{\mathrm{n}} \mathrm{t}_{1}\right)$. The two solutions of this equation are $\mathrm{e}_{\mathrm{n} 1}=0.158 / \mathrm{t}_{1}$ and $\mathrm{e}_{\mathrm{n} 5}=1.921 / \mathrm{t}_{1}$. In the same way, the emission speeds $\mathrm{e}_{\mathrm{n} 2}$ and $e_{n 4}$ are then solutions of the relation $\Delta C=-3 A_{\max } / 4=-3 A / 16$, which gives us the equation $x^{2}-x+3 / 16=0$. The two solutions of this equation are $\mathrm{e}_{\mathrm{n} 2}=0.288 / \mathrm{t}_{1}$ and $\mathrm{e}_{\mathrm{n} 4}=1.386 / \mathrm{t}_{1}$

In summary, all five emission speeds obtained are $\mathrm{e}_{\mathrm{n} 1}=0.158 / \mathrm{t}_{1}, \mathrm{e}_{\mathrm{n} 2}=0.288 / \mathrm{t}_{1}, \mathrm{e}_{\mathrm{n} 3}=0.693 / \mathrm{t}_{1}, \mathrm{e}_{\mathrm{n} 4}=1.386 / \mathrm{t}_{1}$, and $\mathrm{e}_{\mathrm{n} 5}=1.921 / \mathrm{t}_{1}$

The different pairs $\left(\mathrm{e}_{\mathrm{ni}}, \mathrm{T}\right)$ obtained make to draw the line of Arrhenius.

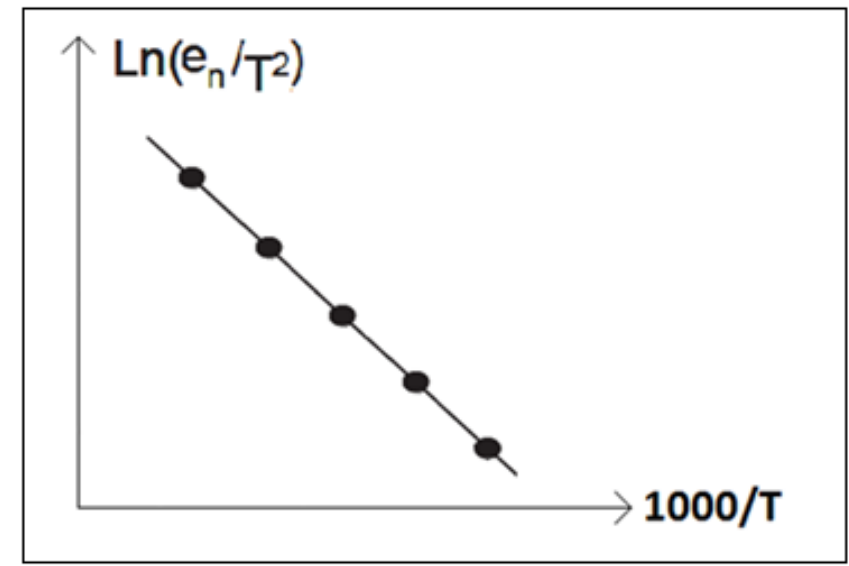

FIGURE 6. The Arrhenius line by the five-points method

\section{EXPERIMENTAL}

The I(V) and $\mathrm{C}(\mathrm{V})$ measurements are a indirect results, based on a models of the defects in the photovoltaic modules. To have direct characterizations, we extended these studies by the DLTS spectroscopy.

In this experiment, we use commercial solar modules $4 \times 4$ (16 cells) $2 \mathrm{~V}, 60 \mathrm{~mA}$ and $0.12 \mathrm{~W}$, collected from the local consumer market. The DLTS was measured in dark condition for different temperatures levels between 140-291K, before and after stresses (electrical, thermal) [4, 6] by adjusting $10 \mathrm{~min}$ for each dose of stress. 
In stressed phase, the electric stress was created in modules by injecting in dark, an inverse electric current equal to $20 \mathrm{~mA}, 30 \mathrm{~mA}$. We have applied this procedure at various fixed temperature levels equal to $50^{\circ} \mathrm{C}, 75^{\circ} \mathrm{C}$. The experimental procedure is presented in figure 7.

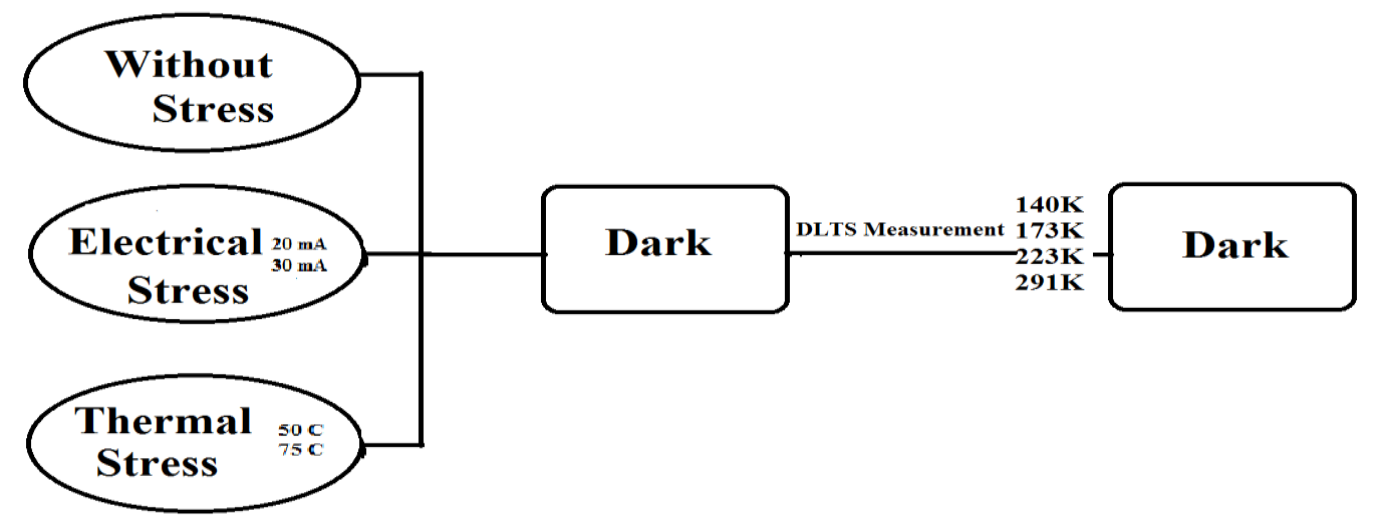

FIGURE 7. Experimental procedure

In DLTS measurment phase, we measure the capacitance as function of temperature in dark condition at different temperature levels between $140 \mathrm{~K}$ and $291 \mathrm{~K}$. During that phase, a negative voltage equal to $-9 \mathrm{~V}$ duration having 2 $\mathrm{ms}$ is applied to the module except a pulse of $100 \mu \mathrm{s}$ duration having an amplitude of $+4 \mathrm{~V}$. This measurement was done for the virgin modules and for the stressed ones.

\section{RESULTS AND DISCUSSIONS}

The shift of the responses $\mathrm{V}(\mathrm{t})$ in the modules was observed in the last paper [17], it is caused because the system photovoltaic module is complex and we have a large surface area but in one cell, the surface of the junction is small. Furthermore, the module also has a very large exchange surface with the metal forming the electrodes and therefore a leakage resistance greater than that of one cell where the current flow is provided by two single wires [17]. After recording the output voltage responses before and after both types of stress as a function of temperature. We can determined the DLTS signals by using the equation 8 .

We have chosen, as detailed in the presentation of Lang's method, a window of the response, $t_{2}-t_{1}$, identical for all the responses $\mathrm{V}(\mathrm{t})$ at the various temperatures and the ratio $\alpha=\mathrm{t}_{2} / \mathrm{t}_{1}$ is equal to 2 . These signals are plotted in figure 8.

$$
\Delta C(p F)=D L T S=\frac{0,2 . \mathrm{Y} \cdot \mathrm{R}}{G} \quad \mathrm{Y}=\mathrm{y} \cdot \mathrm{S}
$$

y: amplitude of Peak (cm); S: Sensivity of Y channel $\left(\frac{\mathrm{mV}}{\mathrm{cm}}\right)$; R: Rang (Pf); G: PRE-AMP GAIN setting. 

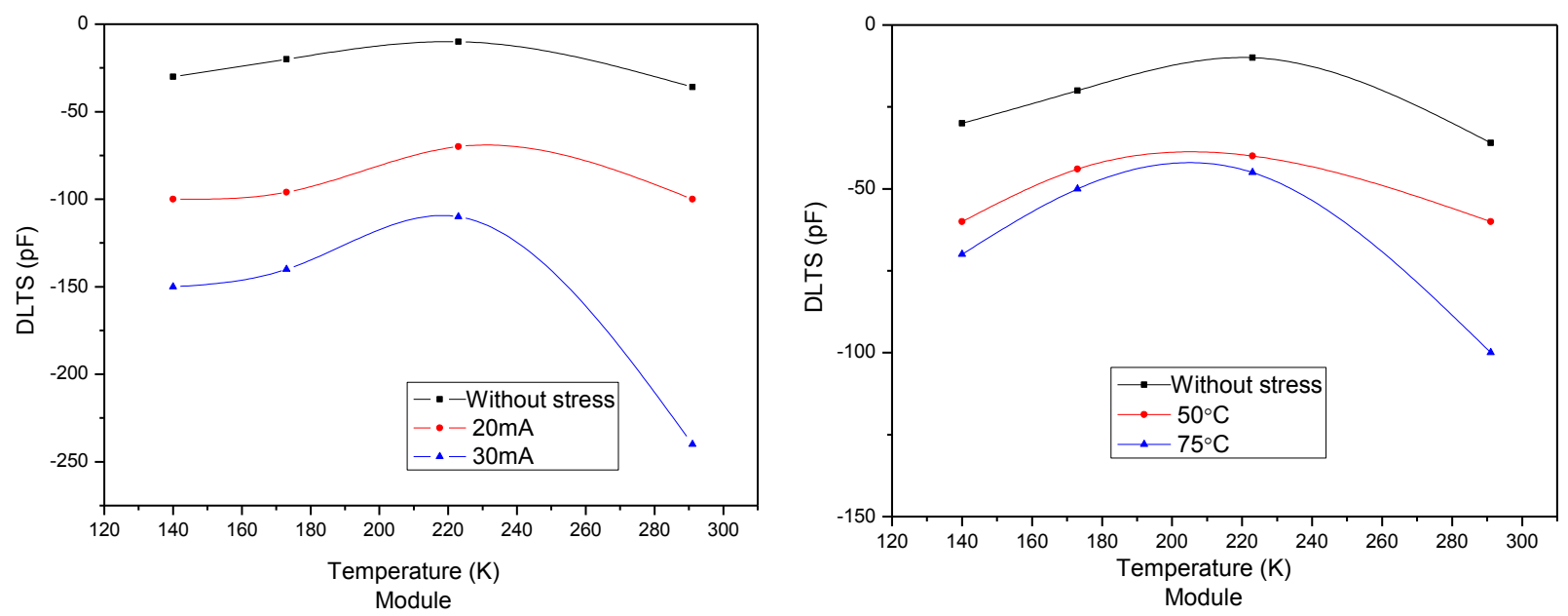

FIGURE 8. DLTS vs. T(K) before and after electric and thermal stresses

From these signals, we applied the five-point method and thus determined five emission speeds $\mathrm{e}_{\mathrm{ni}}$, which are related to five temperatures $T_{i}$, using directly the relations presented previously also in the case we have chosen $(\alpha=2)$. The pairs $\left(e_{n i}, T_{i}\right)$ made it possible to draw Arrhenius lines, shown in figure 9 , from which we can calculate the defect parameters. [18]
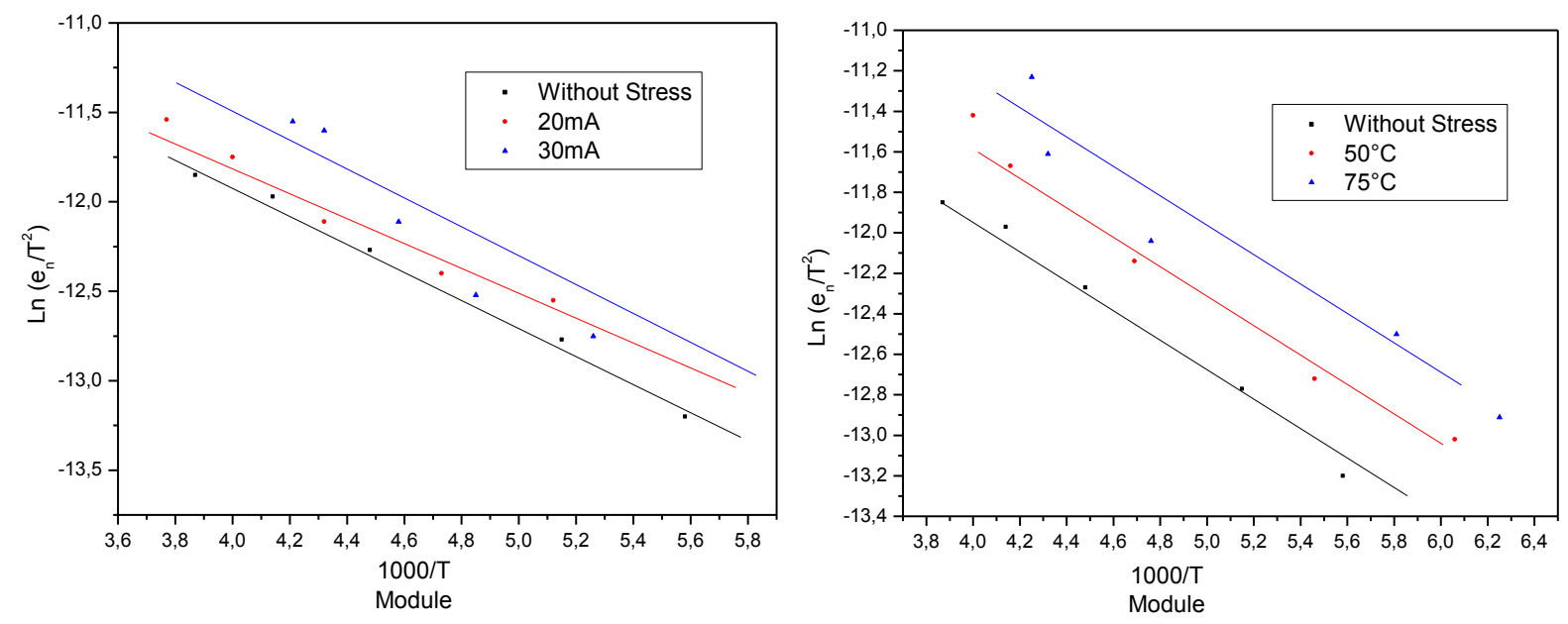

FIGURE 9. The Arrhenius line before and after both type of stress

By calculating the slope of the Arrhenius line and the intersection with the ordinate axis, we obtained the activation energies and the cross sections of the defect for each types of stress. Without stress, the activation energy obtained is $0.4 \mathrm{eV}$ and the capture section is $6.3 \times 10^{-17} \mathrm{~cm}^{2}$.The energy of these defects shows that they are located in the lower part of the gap of the semiconductor [19] compared to the initial value of energy ( $\mathrm{E}_{\mathrm{Fp}}$ equal $0.55 \mathrm{eV}$ in silicon). And we can deduce from this great value of the section obtained, that we can capture the defect easely. We show in table 1 the variation of these parameters as a function of the stress.

TABLE 1. The values of the Ea and $\sigma$ before and after both types of stress

\begin{tabular}{|l|cc|}
\hline Stress électrique & $\mathbf{E}_{\mathrm{a}}(\mathbf{e v})$ & $\boldsymbol{\sigma}\left(\mathrm{cm}^{2}\right)$ \\
\hline Sans Stress & 0.4 & $6.3 \times 10^{-17}$ \\
\hline
\end{tabular}




\begin{tabular}{|l|ll|}
\hline $\mathbf{2 0 m A}$ & 0.71 & $5 \times 10^{-17}$ \\
\hline $\mathbf{3 0 m A}$ & 0.8 & $4.2 \times 10^{-17}$ \\
\hline Stress thermique & $\mathbf{E}_{\mathbf{a}}(\mathbf{e v})$ & $\boldsymbol{\sigma}\left(\mathbf{c m}^{\mathbf{2}}\right)$ \\
\hline Sans Stress & 0.4 & $6.3 \times 10^{-17}$ \\
\hline $\mathbf{5 0}^{\circ} \mathbf{C}$ & 0.69 & $4.54 \times 10^{-17}$ \\
\hline $\mathbf{7 5}^{\circ} \mathbf{C}$ & 0.79 & $4 \times 10^{-17}$ \\
\hline
\end{tabular}

We can conclude by the results obtained before and after the electrical and thermal stress that the activation energy of this defect increases beyond $\mathrm{E}_{\mathrm{Fp}}(0.55 \mathrm{eV})$ after stresses. Nevertheless, we find after the comparision between the values of parameters after both stress, that the activation energies and the capture cross sections are very similar.

In the cases without stress, we observe the presence of a main defect in photovoltaic module (located at $0.4 \mathrm{Ev}$ ). This defect has a very large capture section, about $6.3 \times 10^{-17} \mathrm{~cm}^{2}$, because the module is a complex structure composed of cells in series and parallel. The DLTS results and the reality of the appearance and modification of defects in the moduls under stress is confirmed by the fact that the capture section decreases with increasing stress in the module.

We find that the observed defect moves in the deep levels of the forbidden band with the increase of the stress value, whether electrical or thermal and passes the $\mathrm{E}_{\mathrm{Fp}}$ level. We thus see the direct influence of stress on this main defect, which becomes a deep recombination center, with the reverse current and temperature used to induce the stress, a doubling of activation energy and a decrease of capture section. This phenomenon will induce a greater possibility of capturing the photo-excited electrons, thus decreasing the photovoltaic current and thus the module efficiency.

We can compare these results by the parameters obtained in the last papers [4-8], This low value of shunt resistance and the high value of ideality factor $(A>1)$ when the stress applied, means the increase of the leakage current and a degradation of performance of PV module.

Moreover, these defects can also be attributed to the creation or modification of recombination / generation centers as quantitatively observed by the DLTS measurement, within the limits of the preceding remarks related to the complexity of the structure of the modules. The presence of defects in the modules generally causes malfunctions and deterioration of the electrical characteristics of the photovoltaic modules.

\section{CONCLUSIONS}

Several measurement modes can be use depending on the type of excitation (electrical or optical) and depending of the measured caracterization (voltage, current or capacity).

In this paper, we used a Deep Level Transient Spectroscopy (DLTS) measurement, for the evaluation on the performances of commercial PV modules of the electric and thermal stresses. The DLTS technique is performed for the characterization with high sensitivity of the parameters of defects in the gap of a pure or single semiconductor junction. Here, we used in this experiment a PV modules composed by complex electronic structures.

The main result obtained is that the initially existing defects in the virgin module will have a great activation energy which corresponds to a migration towards the deep levels of the forbidden band and this value increases after the stress applied. By comparing the values of parameters after both stress, the activation energies and the capture cross sections are very similar.

We have investigated the relation between the variation of the parameters of defect and the increase in the diffusion current in the stressed modules, as observed by the electrical and thermal measurements. All these observations thus confirm the reduction in shunt resistance and the degradation of the performance of the cells having undergone a stress which can go as far as the creation of hot spots and the destruction of the module. This allows us to observe that the aging of the modules is directly related to the stress applied.

These results obtained by the DLTS measurement explain the degradation of the performances of the modules before and after the application of electrical or thermal stresses. These methodologies of study may be applicable for one cell, arrays, or even a farm of arrays creating a photovoltaic solar generator.

\section{REFERENCES}

1. J.P. Colinge and C. A. Colinge. "Physics of semiconductor devices", Kluwer Academic Publisher, 2002. 
2. E. Placzek-Popko, J. Trzmiel, E. Zielony, S. Grzanka, R. Czernecki and T. Suski. "Deep level transient spectroscopy signatures of majority traps in $\mathrm{GaN}$ p-n diodes grown by metal-organic vapor-phase epitaxy technique on GaN substrates", Physica B, Vol. 404, pp.4889-4891, 2009.

3. D.V. Lang "Deep- level transient spectroscopy: A new method to characterize traps in semiconductors", J. Appl.Phys., Vol 45, Issue 7, pp.3023-3032, July 1974.

4. J. Zaraket, M. Aillerie and C. Salame, "Dark and Illuminated Characteristics of Photovoltaic Solar Modules. Part I: Influence of Dark Electrical Stress", AIP-CF 1758, 020016, 2016.

5. J. Zaraket, M. Aillerie and C. Salame, "Dark and Illuminated Characteristics of Photovoltaic Solar Modules. Part II: Influence of Light Electrical Stress", AIP-CF, 1758, 030052, 2016.

6. J. Zaraket, T. Salame, M. Aillerie and C. Salame, "Parameters and Characteristics of PV Solar Modules Under the Influence of Thermal Stresses", AIP-CF, 1814, 020055, 2017.

7. J. Zaraket, M. Aillerie and C. Salame, "Capacitance Evolution of Photovoltaic Solar Modules under the Influence of Electrical Stress", Energy Procedia, Elsevier, 1466-1475, Vol 74, 2015.

8. J. Zaraket, M. Aillerie and C. Salame, "Capacitance evolution of PV solar modules under thermal stress", Energy Procedia, Elsevier 702-708, vol 119, 2017.

9. I. Thurzno, K. Kresakova and J. Jakubovic, "on the interpretation of DLTS spectra of MIS capacitors", Journal of physics D :Applied Ohysics,Vol 15 ,pp 1751-1764, 1982.

10. K. Dmowski and A. Jakubowski, "Sensitivity analysis of bulk traps detection in analog deep-level transient spectroscopy measurement systems with exponentially weighted average", rev.Sci.Instrum, Vol.60, pp106, 1989.

11. N. Abboud, "Titre Etude et caractérisation de structure à base de silicium", Thèse cotutelle entre l'université libanaise (Liban) et l'université de Montpellier II (France), 2011.

12. A. Neemgeer, M. Burgelman, "Numerical modelling of ac-characteristics of CdTe and CIS solar cells", 25th IEEE photovoltaic specialist conference, Washington, D.C. 901-904, 1996.

13. Y. Xu, Titre "Fabrication et caractérisation des films CuInGaSe2 par pulvérisation cathodique Etude des défauts par la spectroscopie des pièges profonds par la charge", thèse à université de Nantes faculté des sciences et des techniques, 2014.

14. P. Léveque "Caractérisation des matériaux et des composants", Cours Master 2 MNE,Université Louis paster, UFR Sciences Physiques.

15. M. El Bouabdellati, "Etude par spectroscopie DLTS des structures formees sur ino(n) par oxydation plasma", thèse à l'université de Lorraine, 20 DEC 1994.

16. P. Arnolda, "La création de défauts de déplacements atomiques dans le silicium et son impact sur les composants électroniques à applications spatiales", thèse à l'université de Toulouse, 2011.

17. J. Zaraket, M. Aillerie, C. Salame, and E. Losson "Output Voltage Changes in PV Solar Modules after Electrical and Thermal Stresses. Experimental Analysis.” Energy Procedia, Elsevier, 1404-1411, Vol 157, 2019

18. E. Losson, "Méthodes d'exploitation des transitoires de capacité en spectroscopie des niveaux profonds (DLTS) Application a des jonctions Au-InP”, thèse à l'université de Lorraine ,1992.

19. O. Haas, titre "Étude des défauts électriquement actifs dans les cellules solaires organiques", thèse à université de Nantes faculté des sciences et des techniques, 2012. 